SECTION 31. Economic research, finance, innovation.

Anatoly Aleksandrovich Naumov

Docent, Candidate of Technical Sciences,

Center of Applied Mathematical Research, Novosibirsk, Russia,

E-mail: $\underline{\text { a_a_naumov@mail.ru }}$

\title{
ANALYSIS OF INVEST PROJECTS EFFICIENCY CRITERIA
}

Abstract: The paper discusses classical and new invest projects efficiency criteria. models.

Keywords: Invest projects, efficiency criteria, NPV, NFV, IRR, project optimization,

\section{УДК 336.761: 336.748}

\section{АНАЛИЗ КРИТЕРИЕВ ЭФФЕКТИВНОСТИ ИНВЕСТИЦИОННЫХ ПРОЕКТОВ}

Аннотация: В работе приведены результаты анализа классических и новых критериев эффективности инвестиционных проектов.

Ключевые слова: Инвестиционные проекты, критерии эффективности, NPV, NFV, IRR, оптимизация проектов, модели.

Классические показатели эффективности инвестиционных проектов и их проблемы. Проблемы классических показателей хорошо известны $[1 ; 2 ; 3]$. Напомним основные из них. Так, показатель $N P V$ характеризует приведенный к началу жизни проектов доход. Смысл такого показателя не совсем ясен по нескольким причинам. Вопервых, обычно доход по проектам получают по их завершению. Во-вторых, при мониторинге проектов значение такого показателя придется пересчитывать на момент времени (начала жизни проектов), который, возможно, уже давно пройден. Как выбирать ставку дисконтирования для такого показателя? Почему проводится дисконтирование тех элементов входного потока проектов, которые должны появиться (проявить себя) в проектах только к моментам времени их использования и до этого в проектах никак не фигурируют? Средства выходного потока проектов могут быть задействованы как в рамках анализируемого проекта, так и во внешних для него проектах. Как это обстоятельство учитывается расчетной формулой для показателя? Вопросы, вопросы, вопросы... На большую часть из них логика построения показателя $N P V$ вразумительных ответов не дает. К сожалению, не лучше обстоит дело и с показателем IRR (внутренняя норма доходности). Фактически этот показатель позволяет найти (оценить) всего лишь верхнюю границу для ставки дисконтирования, ниже которой значение показателя NPV принимает положительные значения. Никакого отношения к оценке фактической доходности проектов (которая, кстати, формируется на конец жизни проекта) этот показатель не имеет. Тем более что он оценивает всего лишь верхнюю границу ставки дисконтирования (верхнюю границу для внутренней нормы доходности). Такие проблемы у показателя IRR появились в связи с тем, что они были и у показателя (NPV), на основе которого он построен, и они передались ему, так сказать, «по наследству». Можно назвать еще несколько показателей, построенных на основе показателя $N P V$ и потому получивших от него проблемы.

Функциональная зависимость показателей и задачи оптимизации инвестиционных проектов. Хорошо известно, что поскольку «родителем» для большинства классических показателей эффективности инвестиционных проектов является показатель $N P V$, то, во-первых, этот показатель передал свои слабые стороны (проблемы, алогизмы) «дочерним» показателям, а во-вторых, получилось так, что исходный показатель 
и полученные на его основе новые стали функционально зависимыми. Это следует учитывать при построении оптимизационных моделей для проведения анализа проектов и при нахождении решений в соответствии с этими моделями [4; 5]. Особенно такая функциональная зависимость показателей дает о себе знать (проявляет себя) при построении многокритериальных (векторных) оптимизационных задач. В этом случае целевые функции таких задач становятся зависимыми. Некоторые из них могут быть опущены, а оптимизационные задачи сведены к однокритериальным (скалярным). Иногда решение для таких задач находится в аналитическом виде без использования специальных программных средств и вычислительной техники [6; 7].

Упрощение задач оптимизации инвестиционных проектов. В связи с отмеченными выше обстоятельствами (функциональной зависимостью показателей и проблемами самих показателей), следует аккуратно подходить как к задаче построения оптимизационных задач для анализа проектов, так и к выбору методов их решения. Так, например, в качестве независимых переменных в оптимизационных моделях не рекомендуется использовать без введения дополнительных условий переменные для значений входных (инвестиций) и выходных (доходов) потоков инвестиционных проектов. Хорошо известно, что изменения значений элементов входных потоков (затрат на приобретение оборудования, на проведение работ и т.д.) приведут к изменениям и элементов выходных потоков (себестоимость продукции и т.д.) [6; 7; 8]. Тем более нельзя производить оптимизацию проектов по таким параметрам (переменным), как ставка дисконтирования, срок окупаемости проектов и т.д. Однако это не означает, что, например, срок окупаемости не может быть использован в качестве одного из критериев в оптимизационных задачах и, например, участвовать в качестве одного из их ограничений таких задач. Ситуацию при решении многокритериальных задач может усугубить использование методов, основанных на идее нормализации критериев. Как было показано, такие методы являются неустойчивыми (чувствительными) к изменению пассивных ограничений оптимизационных задач. Кстати, таким свойством не обладает ни один из хорошо известных и распространенных на практике методов многокритериальной оптимизации [8; 9].

Дискретность оптимизационных задач. Как правило, оптимизационные задачи для проектов носят дискретный характер. Это объясняется теми обстоятельствами, например, что варьирование многих переменных этих задач (таких, как цены на ресурсы, цены на произведенную продукцию, поставщики ресурсов и т.д.) приходится проводить, учитывая их дискретный характер. Кроме этого, часто на практике приходится анализировать несколько сценариев развития проектов и выбирать среди множества таких сценариев (вариантов) лучший сценарий. Задача уже по своей сути является дискретной, а метод ее решения - метод перебора вариантов (возможно, направленного перебора) [9;10; $11]$.

Эффективность и риски инвестиционных проектов. При анализе и моделировании проектов необходимо учитывать их риски. Причем, риски необходимо анализировать совместно с основными показателями эффективности проектов, т.е. в парах «показатель эффективности - риск». На наш взгляд, нельзя ставить задачу управления рисками проектов (например, их снижения, минимизации) без учета показателей эффективности. Это объясняется достаточно просто. Изменяя (например, уменьшая) значения рисков, будут изменены и значения показателей эффективности и наоборот. Поэтому наряду с показателями эффективности в модель (например, оптимизационную) для анализа проектов должны быть включены и оценки рисков [1].

Выводы. При анализе инвестиционных проектов следует аккуратно подходить к решению задач выбора показателей эффективности и оцениванию их рисков, построению оптимизационных моделей и выбора методов для их решения. Использование на практике показателей с неясным (не совсем понятным) экономическим смыслом может привести 
(даже при использовании хороших оптимизационных алгоритмов) к результатам столь же неясным и сомнительным, использование которых для реальных проектов может значительно изменить истинную картину относительно эффективности проектов и повысить риски, связанные с их реализацией и последующим продвижением.

\section{References:}

1. Наумов А.А. Методы анализа и синтеза инвестиционных проектов. Эффективность, риски, управление: Монография. - LAP LAMBERT Academic Publishing, 2013. - 356 с.

2. Наумов А.А., Крюков С.В. К проблемам одной модели управления совместными инвестиционными проектами// Финансовая аналитика: проблемы и решения, 2013, № 40 (178), C. 53-56.

3. Наумов А.А. К вопросу о точности оценки дисконтированного срока окупаемости инвестиционного проекта// Финансовая аналитика: проблемы и решения, 2013, № 44 (182), C. 25-28.

4. Наумов А.А. К вопросу об упрощении двух задач оптимизации инвестиций// Финансовая аналитика: проблемы и решения, 2013, № 46 (184), С. 26-30.

5. Наумов А.А. Использование метода детализации финансовых потоков в задачах оценивания эффективности проектов// Финансовая аналитика: проблемы и решения, 2013, № 48 (186), С. 35-41.

6. Наумов А.А. Оценивание эффективности интегрированных проектов// Финансовая аналитика: проблемы и решения, 2014, № 8 (194), С. 36-43.

7. Жанатауов С.У., Наумов А.А. К задаче оценивания частных эффектов интегрированных бизнес-процессов// Вестник Алматинского гуманитарнотехнического университета, 2013, № 2 (12), С. 83-90.

8. Даулетбаков Б.Д., Наумов А.А. Методика определения эффективности инвестиций в инновационные проекты// В кн.: Инновационный тренд экономики Казахстана. Под общей ред. Р.К. Сагиевой. - Алматы: Казак университеті, 2013, с. 99-104.

9. Наумов А.А. Математические модели системы управления продвижением ITпродуктов банка// Theoretical\&Applied Science, Materials of the International Scientific Practical Conference «Theoretical Research», 30.07.2013, Stuttgart, Germany, 2013, №7 (3), C. 95-102.

10. Наумов А.А. К задаче оценивания эффективности и управления продвижением линейки IT- продуктов// Theoretical\&Applied Science, Materials of the ISPC «Theory and Practice», 30.08.2013, Munich, Germany, 2013, № 8 (4), C. 90-92.

11. Список трудов [Электронный ресурс] URL: https://sites.google.com/site/anatolynaumov2011/home/spisok-trudov-list-of-papers (Дата доступа 27.06.14)

12. Научные заметки [Электронный pecypc] URL: https://sites.google.com/site/anatolynaumov2013/home/naucnye-zametki-scientific-notes (Дата доступа 27.06.14) 